\title{
Iron(II) Spin-Crossover Complexes in Ultrathin Films: Electronic Structure and Spin-State Switching by Visible and Vacuum-UV Light**
}

\author{
E. Ludwig, H. Naggert, M. Kalläne, S. Rohlf, E. Kröger, A. Bannwarth, A. Quer, K. Rossnagel, \\ L. Kipp, * and F. Tuczek*
}

\begin{abstract}
The electronic structure of the iron(II) spin crossover complex $\left[\mathrm{Fe}\left(\mathrm{H}_{2} \mathrm{bpz}\right)_{2}(\right.$ phen $\left.)\right]$ deposited as an ultrathin film on $A u(111)$ is determined by means of $U V$-photoelectron spectroscopy (UPS) in the high-spin and in the low-spin state. This also allows monitoring the thermal as well as photoinduced spin transition in this system. Moreover, the complex is excited to the metastable high-spin state by irradiation with vacuum-UV light. Relaxation rates after photoexcitation are determined as a function of temperature. They exhibit a transition from thermally activated to tunneling behavior and are two orders of magnitude higher than in the bulk material.
\end{abstract}

Miniaturization of electronic devices is an important driving force towards reducing functional elements to the size of single molecules. A model system of functional molecules with potential applications in nano-electronics and spintronics, the electrical, magnetic, structural, or optical properties of which can be manipulated by external stimuli, are spin crossover (SCO) complexes, the largest number of examples being available for $\mathrm{Fe}^{\mathrm{II}}$. Depending on the nature and field strength of the surrounding ligands the central iron ion can exist in two different electronic configurations, "low spin" (LS, $S=0)$ and "high spin" (HS, $S=2) .^{[1-4]}$

Herein, the $\mathrm{Fe}^{\mathrm{II}}$ complex $\left[\mathrm{Fe}\left(\mathrm{H}_{2} \mathrm{bpz}\right)_{2}(\right.$ phen $\left.)\right]$ is investigated (compound $\mathbf{1} ; \mathrm{H}_{2} \mathrm{bpz}=1$,2-bis(pyrazolyl)borate, phen = 1,10'-phenanthroline; see Figure 1, top). This compound undergoes a thermal spin transition between HS and LS at $T_{1 / 2} \approx 164 \mathrm{~K}$ (Figure 1, bottom). Below $T_{\text {LIESST }}=43 \mathrm{~K}$, the complex can be switched to a metastable HS (mHS) state by

[*] E. Ludwig, Dr. M. Kalläne, S. Rohlf, E. Kröger, A. Quer,

Dr. K. Rossnagel, Prof. Dr. L. Kipp

Institut für Experimentelle und Angewandte Physik

Christian-Albrechts-Universität zu Kiel

24098 Kiel (Germany)

E-mail: kipp@physik.uni-kiel.de

Homepage: www.ieap.uni-kiel.de/surface/ag-kipp

H. Naggert, Dr. A. Bannwarth, Prof. Dr. F. Tuczek

Institut für Anorganische Chemie

Christian-Albrechts-Universität zu Kiel

24098 Kiel (Germany)

E-mail: ftuczek@ac.uni-kiel.de

Homepage: www.ac.uni-kiel.de/tuczek

[**: T. Riedel and the Petra III staff at DESY are thanked for technical assistance as well as Dr. L. Carella and Prof. Dr. E. Rentschler (Mainz) for bulk magnetic measurements under light irradiation. This work was supported by DFG via SFB 677 and BMBF via project No. 05K10FK1.

9 Supporting information for this article is available on the WWW under http://dx.doi.org/10.1002/anie.201307968.
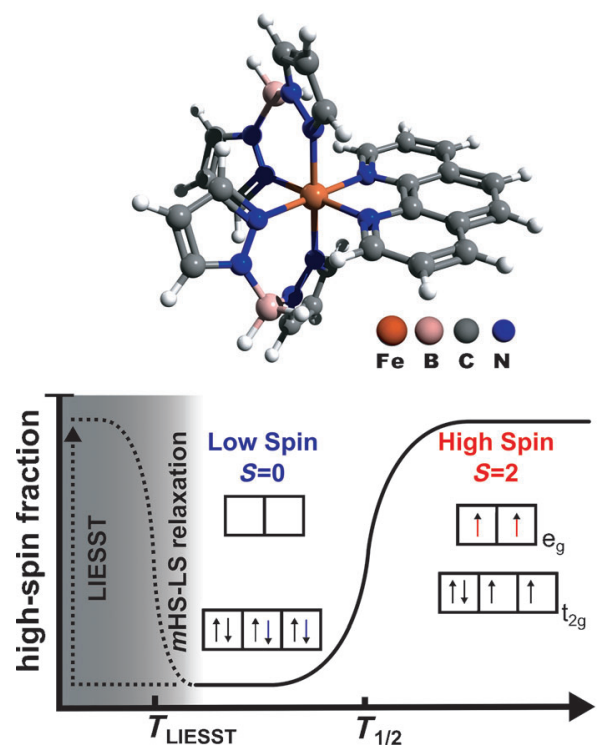

Figure 1. Representation of $\mathbf{1}$ and $\mathrm{Fe}$ " high-spin fraction versus temperature with LIESST effect; the configuration of the Fe d electrons is indicated.

irradiation with $532 \mathrm{~nm}$ light. ${ }^{[5]}$ This effect is known as lightinduced excited spin-state trapping (LIESST). ${ }^{[6-8]}$ The lifetime of the metastable HS state is very long at temperatures well below $T_{\text {LIESST }}$ but strongly decreases with increasing temperature, eventually leading to relaxation to the LS ground state at $T=T_{\text {LIESST }}$ (Figure 1, bottom). ${ }^{[2]}$

In bulk materials, the LIESST effect has been well investigated..$^{[9,10]}$ Successful vacuum deposition of $\mathbf{1}$ and the related complex $\left[\mathrm{Fe}\left(\mathrm{H}_{2} \mathrm{bpz}\right)_{2}\right.$ (bipy)] (2; bipy $=2,2^{\prime}$-bipyridine $)^{[11,12]}$ has opened up the possibility to study this effect in solvent-free high-quality films, using methods that require ultra-high vacuum conditions, such as ultraviolet ${ }^{[13]}$ and X-ray photoelectron or inverse photoemission spectroscopy. ${ }^{[14]}$ In recent studies, thin films of $\mathbf{1}$ and $\mathbf{2}$ have also been investigated with scanning tunneling microscopy ${ }^{[15-17]}$ as well as X-ray absorption spectroscopy, ${ }^{[18-20]}$ and evidence for electron-induced spin-state switching has been provided for 1. $^{[15,16]}$ Detailed studies of the valence electronic structure of $\mathbf{1}$ and $\mathbf{2}$ are still missing, however. The same applies to the dynamics of the photoexcited spin state exhibited by these systems in ultrathin films. A recent study has shown that the spin transition temperature and the relaxation dynamics of the metastable $\mathrm{HS}$ state of $\mathrm{Fe}^{\mathrm{II}}$ can vary greatly from crystalline to nanocrystalline and amorphous samples. ${ }^{[21]}$ 
Herein we present an ultraviolet photoelectron spectroscopy (UPS) study of the electronic structure and the lightinduced switching behavior of $\mathbf{1}$ in vacuum-deposited ultrathin films. The dynamic population of the metastable HS state is investigated under permanent irradiation for different laser intensities to determine mHS-to-LS relaxation rates at temperatures below, around, and well above $T_{\text {LIESST }}(\mathbf{1})=$ $43 \mathrm{~K}$.

The electronic structure of a thin layer of $\mathbf{1}$ determined by UPS, and a comparison to spectra obtained from DFT calculations, is shown in Figure 2 (for experimental details,

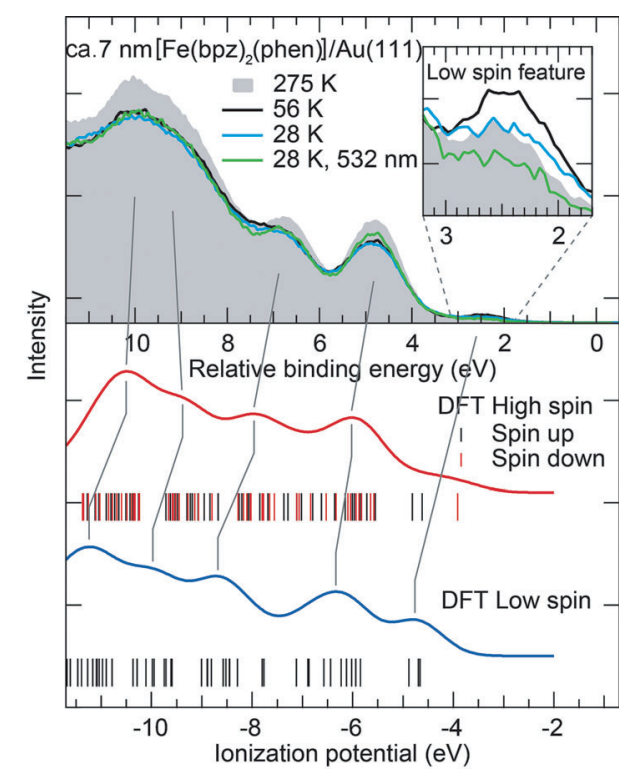

Figure 2. Top: Valence band structure of a circa $7 \mathrm{~nm}$-thick layer of 1 on $\mathrm{Au}(111)$ at $275 \mathrm{~K}$ (filled gray area), $56 \mathrm{~K}$ (black line), $28 \mathrm{~K}$ (blue line), and $28 \mathrm{~K}$ with additional irradiation with $532 \mathrm{~nm}$ (green line). Photon energy for photoemission was $21.22 \mathrm{eV}$. The inset emphasizes the feature at a binding energy of $2.4 \mathrm{eV}$. Bottom: Vertical bars illustrate the ionization energies results of molecular orbitals as obtained from the DFT calculations for the LS and HS case. Spectra composed from the DFT results are shown as blue (LS) and red lines (HS). Dark gray lines serve as guides to the eye to roughly assign features of the theoretical data to the measured valence band structure.

see the Supporting Information). The upper part of Figure 2 shows the valence electronic structure of an about $7 \mathrm{~nm}$-thick layer of 1 on $\mathrm{Au}(111)$, corresponding to 5-6 molecular layers, if a size of $1.4 \mathrm{~nm} \times 0.76 \mathrm{~nm}$ of the individual molecule is assumed. ${ }^{[16]}$ The spectra are composed of several broad features at binding energies of $2.4 \mathrm{eV}, 4.8 \mathrm{eV}, 6.8 \mathrm{eV}$, and a very broad feature centered at about $9.6 \mathrm{eV}$. Comparison of spectra at near room temperature, at $56 \mathrm{~K}$, and at $28 \mathrm{~K}$ shows no significant changes of the overall electronic structure. Additional irradiation with $532 \mathrm{~nm}$ at $28 \mathrm{~K}$ seems to have little influence as well. Detailed investigation of the feature at a binding energy of about $2.4 \mathrm{eV}$, however, reveals an intensity dependence on temperature as well as on $532 \mathrm{~nm}$ irradiation (shown in the inset of Figure 2; see below). For better visibility and to account for sample charging, the energy axis of the spectra has been calibrated to the binding energy of the feature located at $4.8 \mathrm{eV}$.

The bottom part of Figure 2 shows the spectra derived from the results of the DFT calculations at the BP86/tzvp level. ${ }^{[15,22]}$ The calculated ionization energies are indicated as vertical lines below the respective spectra. The theoretical spectra were obtained by assigning a Gaussian profile of equal intensity and width to each transition. Polarization- and matrix-element related effects were not taken into account. The overall shape of the calculated spectra matches the photoemission very well. A rough correlation between experimental and theoretical spectral features is indicated by dark gray lines.

A clear difference of the theoretical spectra between the two spin states can be observed at ionization energies of about $4.5 \mathrm{eV}$. In the LS state, the three doubly occupied $t_{2 g}$ orbitals give rise to a distinct feature at $4.5-5 \mathrm{eV}$, which will be referred to as a "low-spin feature". Owing to the spin polarization effect, the dorbitals are distributed over a much larger energy range in the HS state, and in the spectral region of the low-spin feature much less intensity is predicted. Experimentally, cooling of the sample from $275 \mathrm{~K}$ to $56 \mathrm{~K}$ is accompanied by an intensity increase of the LS feature (Figure 2 inset). Further cooling to $28 \mathrm{~K}$, however, leads to an intensity decrease, and irradiation with $532 \mathrm{~nm}$ light at $28 \mathrm{~K}$ further decreases the intensity of this feature.

A more detailed investigation of these phenomena is shown in Figure 3, where the intensity of the LS feature is

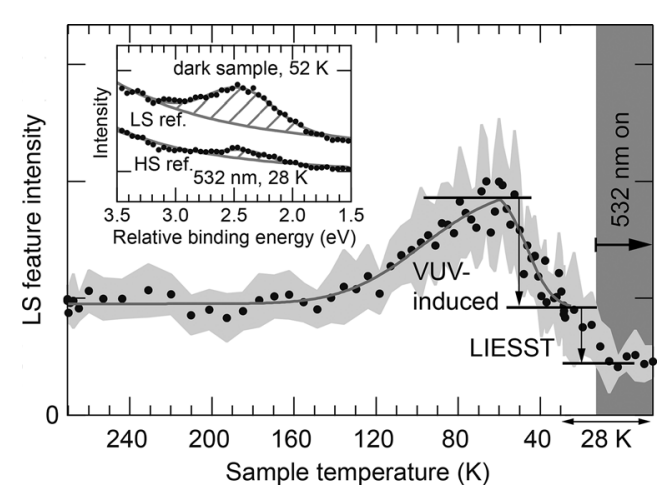

Figure 3. Evolution of the low-spin feature intensity during sample cooling, as obtained from a Gaussian fit function. The Gaussian fit component is illustrated in the inset for spectra recorded at $52 \mathrm{~K}$ und at $28 \mathrm{~K}$ with illumination as a shaded area. The gray shaded area indicates the error margins obtained from the fit procedure. Dark gray lines illustrate the overall intensity evolution. Intensity variations due to the VUV-induced and LIESST effects are indicated by arrows. The shaded area on the right indicates measurements under irradiation with $532 \mathrm{~nm}$.

plotted versus the sample temperature during sample cooling and additional irradiation of the sample with $532 \mathrm{~nm}$ at $28 \mathrm{~K}$. A fit function consisting of an exponentially decaying background and a Gaussian profile is used to determine the temperature dependence of the feature intensity. The inset in Figure 3 illustrates the Gaussian fit component as a shaded area. 
Upon cooling the sample, we first observe an increase of the LS feature starting at about $180 \mathrm{~K}$ down to about $60 \mathrm{~K}$. This behavior reflects a thermal spin transition from the HS to the LS state. Further lowering of the temperature then leads to an intensity decrease to about room temperature level with a transition temperature of about $50 \mathrm{~K}$, indicating a population of the mHS state. Presumably, this transition is induced by the vacuum-UV (VUV) irradiation of the sample, similar to the optical excitation of $\mathbf{1}$ to the mHS state, which occurs at about the same temperature. ${ }^{[5]}$ The VUV-induced process, however, does not lead to full conversion from the LS to the HS state, as additional irradiation of the sample at $28 \mathrm{~K}$ with the $532 \mathrm{~nm}$ laser causes a further intensity decrease of the LS feature, as indicated by the dark gray area in Figure 3. We assume that now all molecules with active centers are in the HS state (low-spin fraction $\gamma_{\mathrm{LS}}=0$ ). Importantly, the thermal spin transition in our film of $\mathbf{1}$ appears to occur at a much lower temperature than in the bulk material $(165 \mathrm{~K}),{ }^{[5,9]}$ and the spin crossover behavior is much more gradual. This seems to agree with the rule that the spin transition generally is less steep and occurs at lower temperatures for nanocrystalline or amorphous samples as compared to the crystalline bulk material. ${ }^{[21]}$

The observation that the intensity of the LS feature at $28 \mathrm{~K}$ is lowered by illumination of the sample with laser light prompted us to explore the influence of a continuous laser irradiation at $532 \mathrm{~nm}$ on the spin equilibrium in ultrathin films of $\mathbf{1}$ in more detail. The results of these measurements are shown in Figure 4 where the low-spin fraction $\gamma_{\mathrm{LS}}$ observed

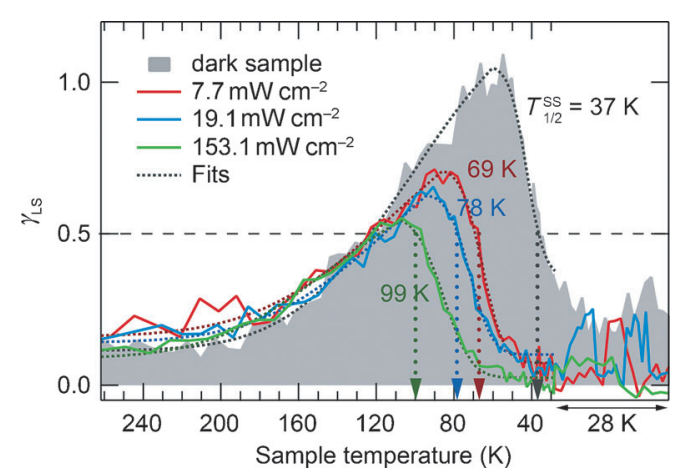

Figure 4. Low-spin fraction versus temperature as a function of permanent irradiation with varying intensities of $532 \mathrm{~nm}$ light. Steadystate spin transition temperatures $T_{1 / 2}$ ss are indicated by dotted vertical arrows. Gray shaded areas illustrate measurements without additional irradiation.

under irradiation of the sample with different laser intensities is plotted versus the temperature. The laser was also switched off for short periods at $28 \mathrm{~K}$. A representative spin transition curve without laser irradiation is given as a reference (gray filled trace in Figure 4). The intensity of the LS feature was determined as shown in Figure 3; for the determination of the LS fraction, the residual intensity of the LS feature in the high spin state was accounted for (see the Supporting Information). The temperatures at which a stationary population of the mHS state of $50 \%$ occurs $\left(\gamma_{\mathrm{LS}}=0.5\right)$ are defined as steady-state spin transition temperatures $T_{1 / 2}$ SS $(\mathrm{SS}=$ steady state). Without any additional irradiation a value of $T_{1 / 2} \mathrm{ss}=$ $37 \mathrm{~K}$ is observed. Importantly, permanent irradiation of the sample with $532 \mathrm{~nm}$ light shifts $T_{1 / 2}^{\text {ss }}$ to higher temperatures, depending on the intensity of the irradiation. Averaging spin transition temperatures obtained for several measurements with the same laser power density results in $69 \mathrm{~K}$ for $7.7 \mathrm{~mW} \mathrm{~cm}^{-2}, \quad 78 \mathrm{~K}$ for $19.1 \mathrm{~mW} \mathrm{~cm}^{-2}$, and $99 \mathrm{~K}$ for $153.1 \mathrm{~mW} \mathrm{~cm}^{-2}$ (Table 1). A plot of the steady-state transition

Table 1: Steady-state spin transition temperatures and light-induced SCO excitation rates calculated from the laser intensities.

\begin{tabular}{ccl}
\hline $\begin{array}{c}\text { Spin transition temperature } \\
T_{1 / 2} \text { ss }[\mathrm{K}]\end{array}$ & $\begin{array}{l}\text { Laser intensity } \\
{\left[\mathrm{mW} \mathrm{cm}{ }^{-2}\right]}\end{array}$ & $\begin{array}{l}\text { Excitation rate } \\
k_{\mathrm{ex}}\left[\mathrm{s}^{-1}\right]\end{array}$ \\
\hline 37 & 0 (VUV exc. only) & $5.8 \times 10^{-3}$ \\
69 & 7.7 & 0.3 \\
78 & 19.1 & 0.7 \\
99 & 153.1 & 5.6 \\
\hline
\end{tabular}

temperature $T_{1 / 2}$ ss versus the laser power indicates a logarithmic dependence (Supporting Information, Figure S1). Note that the signal-to-noise ratio decreases for low laser power densities. When switching off the laser at low temperatures, the intensity of the low-spin feature relaxes to about room temperature level within the recording time of one photoelectron spectrum (about $130 \mathrm{~s}$ ). Resuming the laser irradiation leads to an intensity decrease again, as observed before.

The shift of the spin-transition temperatures under permanent laser irradiation can be explained by a steadystate population of the metastable state above $T_{\text {LIESST }}$ owing to a high photon flux. A light-induced shift of $T_{\text {LIESST }}$ towards higher temperature has previously been observed for crystalline $\mathrm{Fe}^{\mathrm{II}}$ spin crossover complexes in conjunction with a lightinduced thermal hysteresis (LITH). ${ }^{[25,26]}$ Susceptibility measurements under permanent irradiation show that this effect exists for bulk samples of $\mathbf{1}$ as well (Supporting Information, Figure S2). In the thin film of $\mathbf{1}$, however, no hysteresis is observed, as curves of the LS feature intensity versus $T$ obtained upon heating the sample are similar to those determined in the cooling mode (Supporting Information, Figure S3).

In Table 1, excitation rates $k_{\text {ex }}$ calculated from the rate of incident photons are also shown. These values are calculated from a cross section of $1.37 \times 10^{-17} \mathrm{~cm}^{2}$ for excitation with $532 \mathrm{~nm} \mathrm{light}^{[12]}$ and a quantum efficiency of 1.0 for the lightinduced population of the metastable HS state (see the Supporting Information). ${ }^{[27]}$ The steady-state high-spin fraction under continuous irradiation as a function of $k_{\mathrm{ex}}$ and the $\mathrm{HS} \rightarrow \mathrm{LS}$ relaxation rate $k_{\mathrm{HL}}$ is given by Equation (1): ${ }^{[21]}$

$\gamma_{\mathrm{HS}}^{\mathrm{SS}}=1-\gamma_{\mathrm{LS}}^{\mathrm{SS}}=\frac{k_{\mathrm{ex}}}{k_{\mathrm{ex}}+k_{\mathrm{HL}}}$

Therefore $k_{\mathrm{HL}} \approx k_{\mathrm{ex}}$ at $T_{1 / 2}$ ss, where $\gamma_{\mathrm{HS}}{ }^{\mathrm{sS}}=\gamma_{\mathrm{LS}} \mathrm{ss} \approx 0.5$. The $\mathrm{LS} \rightarrow \mathrm{HS}$ excitation rates $k_{\mathrm{ex}}$ of Table 1 thus can be identified with the $\mathrm{HS} \rightarrow \mathrm{LS}$ relaxation rates $k_{\mathrm{HL}}$. A plot of the relaxation rates $k_{\mathrm{HL}}$ determined this way versus $1 / T$ is shown in Figure 5. 


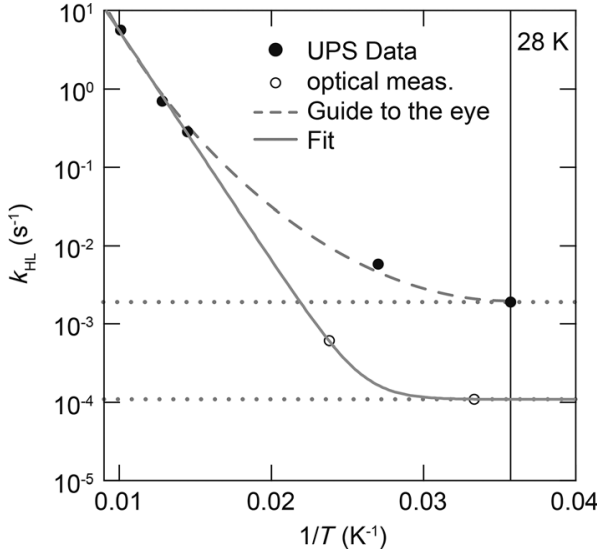

Figure 5. Arrhenius plot of the HS-to-LS relaxation constants obtained from measurements under permanent irradiation. Filled circles show data obtained from UPS measurements, open circles those determined with UV/Vis absorption spectroscopy in a $480 \mathrm{~nm}$ film. The full gray line shows a fit resulting from superposition of a tunneling process and an activated process with $E_{A}=469 \mathrm{~cm}^{-1}$ and $\left.A=4.7 \times 10^{3} \mathrm{~s}^{-1}\right)$. The dashed gray curve serves as guide to the eye. Dotted gray lines emphasize the minimum relaxation rate for the thin film system under VUV irradiation $\left(1.9 \times 10^{-3} \mathrm{~s}^{-1}\right)$ and the $480 \mathrm{~nm}$ film (ca. $10^{-4} \mathrm{~s}^{-1}$ ).

For comparison, relaxation rates derived from optical measurements of a $480 \mathrm{~nm}$-thick film of 1 (Supporting Information, Figure S5) are also given. Importantly, the decay curves obtained for these films after excitation with $532 \mathrm{~nm}$ light exclusively exhibit mono-exponential behavior (Supporting Information, Figure S6), in contrast to those recorded for the bulk material of $\mathbf{1}$, which reflect the presence of cooperative interactions. $^{[5]}$

As evident from Figure 5, the high-temperature relaxation rates derived from UPS on ultrathin films of $\mathbf{1}$ (filled circles) can be described with an Arrhenius behavior, and extrapolation to low temperatures leads to the values determined for the $480 \mathrm{~nm}$ thick film by UV/Vis absorption spectroscopy ( $k_{\mathrm{HL}} \approx 10^{-3}-10^{-4} \mathrm{~s}^{-1}$; open circles). A fit curve simulating a transition from thermally activated to tunneling behavior is given by a gray line, leading to a low-temperature limit for $k_{\mathrm{HL}}$ in vacuum-deposited films of $\mathbf{1}$ on the order of about $10^{-4} \mathrm{~s}^{-1}$. Notably, this value is about one order of magnitude higher than the low-temperature limit of $k_{\mathrm{HL}}$ in the crystalline bulk material of $\mathbf{1}$ (ca. $\left.10^{-5} \mathrm{~s}^{-1}\right){ }^{[5]}$ On the other hand, the relaxation rate in the thin film of $\mathbf{1}$ at $28 \mathrm{~K}$ must be significantly higher than the thick-film value of $10^{-4} \mathrm{~s}^{-1}$, as after switching off irradiation with $532 \mathrm{~nm}$ light (having converted the LS to the HS state to $100 \%$ ) the LS fraction is found to return to a level similar to the room temperature value (ca. 0.25) within the acquisition time of one photoelectron spectrum (130s; Figure 4). Based on a value of $\gamma_{\mathrm{HS}}{ }^{\mathrm{SS}}=0.75$ and the corresponding relaxation kinetics (see the Supporting Information), we conclude that $k_{\mathrm{HL}}=1.9 \times$ $10^{-3} \mathrm{~s}^{-1}$ and $k_{\mathrm{ex}}=5.8 \times 10^{-3} \mathrm{~s}^{-1}$. The given $k_{\mathrm{HL}}$ value can be considered as the lower limit of relaxation rates attainable to the ultrathin films of $\mathbf{1}$ under our experimental conditions (that is, in the presence of continuous VUV irradiation), one and two orders of magnitude higher than in the thick film and in the crystalline sample of $\mathbf{1},{ }^{[5]}$ respectively. The value of $k_{\mathrm{ex}}=$
$5.8 \times 10^{-3} \mathrm{~s}^{-1}$ corresponds to excitation from the LS to the HS state owing to VUV irradiation. This value of $k_{\mathrm{ex}}$ equals $k_{\mathrm{HL}}$ at $T_{1 / 2}{ }^{\mathrm{SS}}=37 \mathrm{~K}$, where a dynamic spin equilibrium with $\gamma_{\mathrm{HS}}^{\mathrm{sS}}=$ 0.5 under continuous VUV irradiation is observed (see Figure 4 "dark sample" and Table 1). The apparently close agreement between this spin-transition temperature and $T_{\text {LIESST }}=43 \mathrm{~K}$ determined for $\mathbf{1}$ after optical excitation in the bulk (see below) is therefore accidental. As a matter of fact, the excitation mechanisms and the relaxation times are quite different for these two cases.

To conclude, ultraviolet photoelectron spectroscopy has been shown to be a suitable method to investigate the electronic structure as well as the thermal and light-induced spin-crossover behavior of $\mathbf{1}$ in ultrathin films on $\mathrm{Au}(111)$. Our results confirm that spin switching of $\mathrm{Fe}^{\mathrm{II}}$ complexes is possible in such systems, but with significantly larger relaxation rates than in the bulk. The photoelectron spectra of a circa $7 \mathrm{~nm}$-thick film of $\mathbf{1}$ can be reproduced with DFT calculations of the complex in the gas phase, indicating that the molecules deposited on the $\mathrm{Au}(111)$ surface are intact. Evaluation of the intensity of the LS feature in the photoelectron spectra yields transition temperatures $T_{1 / 2}$ for the thermal HS-LS spin crossover that are significantly lower than in the bulk (164 K). For the first time, quantitative lightinduced conversion of the low-spin to the metastable highspin state has been achieved for a $\mathrm{Fe}^{\mathrm{II}}$ complex deposited as a thin layer on gold. Furthermore, $\mathrm{mHS} \rightarrow \mathrm{LS}$ relaxation rates have been determined for such a system. Without additional laser irradiation, a VUV-induced excited spin state trapping (VUVIESST) effect is observed; the corresponding steadystate spin transition temperature $T_{1 / 2}{ }^{\text {Ss }}$ is determined to $37 \mathrm{~K}$. Permanent irradiation with a $532 \mathrm{~nm}$ laser shifts $T_{1 / 2}{ }^{\text {ss }}$ toward higher temperatures, which is explained by a competition between (temperature-dependent) $\mathrm{HS} \rightarrow \mathrm{LS}$ relaxation and (power-dependent) LS $\rightarrow$ HS excitation through optical irradiation. The temperature dependence of $k_{\mathrm{HL}}$ shows a transition from Arrhenius behavior to tunneling. A low-temperature limit for the relaxation rates in the thin films systems seems to be a value of about $10^{-3} \mathrm{~s}^{-1}$, which is one to two orders of magnitude higher than in thick films or the bulk.

Population of the metastable high-spin state in the absence of laser illumination is induced by irradiation of the sample with VUV photons of $21.22 \mathrm{eV}$ energy. Similar to the spin-state-trapping effects by exposure to hard and soft X-ray radiation, ${ }^{[24,25]}$ secondary electrons created during the photoemission process can transfer energy to the Fe complex by inelastic scattering processes, leading to population of the metastable high spin state. In our case, however, this effect is much weaker, because the photon flux of a laboratory VUV source is several orders of magnitude lower in comparison to synchrotron sources.

Received: September 10, 2013

Revised: November 20, 2013

Published online: February 12, 2014

Keywords: molecular switches - spin crossover - thin films . ultraviolet photoelectron spectroscopy 
[1] P. Gütlich, H. A. Goodwin, Spin Crossover in Transition-Metal Compounds, Vol. I-III, Springer, Berlin-Heidelberg-New York, 2004.

[2] P. Gütlich, A. Hauser, H. Spiering, Angew. Chem. 1994, 106, 2109-2141; Angew. Chem. Int. Ed. Engl. 1994, 33, 2024-2054.

[3] O. Kahn, C. J. Martinez, Science 1998, 279, 44-48.

[4] T. Mahfoud, G. Molnár, S. Cobo, L. Salmon, C. Thibault, C. Vieu, P. Demont, A. Bousseksou, Appl. Phys. Lett. 2011, 99, 053307.

[5] N. Moliner, L. Salmon, L. Capes, M. C. Muñoz, J.-F. Létard, A Bousseksou, J.-P. Tuchagues, J. J. McGarvey, A. C. Dennis, M. Castro, R. Burriel, J. A. Real, J. Phys. Chem. B 2002, 106, $4276-$ 4283.

[6] S. Decurtins, P. Gütlich, C. P. Köhler, H. Spiering, A. Hauser, Chem. Phys. Lett. 1984, 105, 1-4.

[7] S. Decurtins, P. Gütlich, K. M. Hasselbach, A. Hauser, H. Spiering, Inorg. Chem. 1985, 24, 2174-2178.

[8] A. Hauser, Chem. Phys. Lett. 1986, 124, 543-548.

[9] J. A. Real, A. B. Gaspar, M. C. Muñoz, Dalton Trans. 2005, $2062-2079$

[10] J.-F. Létard, J. Mater. Chem. 2006, 16, 2550-2559.

[11] S. Shi, G. Schmerber, J. Arabski, J.-B. Beaufrand, D. J. Kim, S. Boukari, M. Bowen, N. T. Kemp, N. Viart, G. Rogez, E. Beaurepaire, H. Aubriet, J. Petersen, C. Becker, D. Ruch, Appl. Phys. Lett. 2009, 95, 043303.

[12] H. Naggert, A. Bannwarth, S. Chemnitz, T. von Hofe, E. Quandt, F. Tuczek, Dalton Trans. 2011, 40, 6364-6366.

[13] M. Kamada, K. Takahashi, Y. Doi, K. Fukui, T. Tayagaki, K. Tanaka, Phase Transitions 2002, 75, 847-853.

[14] X. Zhang, T. Palamarciuc, P. Rosa, J.-F. Létard, B. Doudin, Z. Zhang, J. Wang, P. A. Dowben, J. Phys. Chem. C 2012, 116, 23291-23296.

[15] T. G. Gopakumar, F. Matino, H. Naggert, A. Bannwarth, F. Tuczek, R. Berndt, Angew. Chem. 2012, 124, 6367-6371; Angew. Chem. Int. Ed. 2012, 51, 6262-6266.
[16] T. G. Gopakumar, F. Matino, H. Naggert, A. Bannwarth, F. Tuczek, R. Berndt, Angew. Chem. 2013, 125, 3884; Angew. Chem. Int. Ed. 2013, 52, 3796.

[17] T. Miyamachi, M. Gruber, V. Davesne, M. Bowen, S. Boukari, L. Joly, F. Scheurer, G. Rogez, T. K. Yamada, O. Ohresser, E. Beaurepaire, W. Wulfhekel, Nat. Commun. 2012, 3, 938.

[18] B. Warner, J. C. Oberg, T. G. Gill, F. El Hallak, C. F. Hirjibehedin, M. Serri, S. Heutz, M.-A. Arrio, P. Sainctavit, M. Mannini, G. Poneti, R. Sessoli, P. Rosa, J. Phys. Chem. Lett. 2013, 4, $1546-$ 1552.

[19] J.-J. Lee, H.-S. Sheu, C.-R. Lee, J.-M. Chen, J.-F. Lee, C.-C. Wang, C.-H. Huang, Y. Wang, J. Am. Chem. Soc. 2000, 122, $5742-5747$.

[20] M. Bernien, D. Wiedemann, C. F. Hermanns, A. Krüger, D. Rolf, W. Kroener, P. Müller, A. Grohmann, W. Kuch, J. Phys. Chem. Lett. 2012, 3, 3431-3434.

[21] P. Chakraborty, M.-L. Boillot, A. Tissot, A. Hauser, Angew. Chem. 2013, 125, 7279-7282; Angew. Chem. Int. Ed. 2013, 52, $7139-7142$

[22] In Ref. [16], the functional/basis set has erroneously been given as tppsh/tzvp.

[23] D. Collison, C. D. Garner, C. M. McGrath, J. F. W. Mosselmans, M. D. Roper, J. M. W. Seddon, E. Sinn, N. A. Young, J. Chem. Soc. Dalton Trans. 1997, 4371-4376.

[24] G. Vankó, F. Renz, G. Molár, T. Neisius, S. Kárpáti, Angew. Chem. 2007, 119, 5400-5403; Angew. Chem. Int. Ed. 2007, 46, $5306-5309$.

[25] A. Desaix, O. Roubeau, J. Jeftic, J. G. Haasnoot, K. Boukheddaden, E. Codjovi, J. Linarès, M. Noguès, F. Varret, Eur. Phys. J. B 1998, 6, 183-193.

[26] J.-F. Létard, P. Guionneau, L. Rabardel, J. A. K. Howard, A. E. Goeta, D. Chasseau, O. Kahn, Inorg. Chem. 1998, 37, 44324441.

[27] A. Hauser, Top. Curr. Chem. 2004, 234, 155-198. 